\title{
Recomendaciones en el tratamiento de la tuberculosis sensible (Según las Guías ATS/CDC/IDSA. 2016)
}

\author{
MACARENA LAGOS C.*, JUAN CARLOS RODRÍGUEZ D.**, \\ CARLOS PEÑA M.*** y VICTORINO FARGA C.***
}

Guidelines for the treatment of drug-susceptible tuberculosis. According to official ATS, CDC and IDSA Guidelines 2016

During 2016, American Thoracic Society: ATS, Centers for Disease Control and Prevention: CDC and Infectious Disease Society of America: IDSA jointly sponsored the development of Guidelines for the treatment of drug-susceptible tuberculosis using the P-I-C-O (Patient-Intervention-ComparisonOutcome) system to answer nine questions. The preferred regimen for treating adults with drug-susceptible tuberculosis, consider a 2 month intensive phase with isoniazid (INH), rifampin (RIF), pyrazinamide (PZA), and ethambutol (EMB) followed by a continuation phase of 4 months of INH and RIF. For patients who have cavitation on the initial chest radiograph and have positive cultures at completion of 2 months therapy, it is recommended to extend the continuation phase to prevent relapse. EMB can be discontinued as soon as susceptibility to INH and RIF is demonstrated. The guidelines also makes recommendations for the management in different clinical situations as in patients co-infected with HIV and in extrapulmonary tuberculosis. In tuberculous pericarditis no longer is recommended the routinely use of corticosteroids together with the anti TB treatment. In the case of tuberculous meningitis it is recommended the addition of corticosteroids and to extend the continuation phase to 9-12 months.

Key words: Tuberculosis treatment; Antitubercular agents; HIV infection; case management; public health.

\section{Resumen}

Durante el año 2016, la American Thoracic Society: ATS, Centers for Disease Control and Prevention: $C D C$ y la Infectious Disease Society of America: IDSA desarrollaron en conjunto una guía de recomendaciones para el tratamiento de la tuberculosis (TBC) sensible, empleando el sistema P-I$\mathrm{C}-\mathrm{O}$ (Patient-Intervention-Comparison-Outcome) para contestar 9 preguntas. El régimen de terapia farmacológica óptima para el paciente con TBC sensible considera una fase intensiva de 2 meses de duración con isoniacida (H), rifampicina (R), pirazinamida (Z) y etambutol (E) seguida de una fase de continuación por 4 meses de $H$ y $R$. En los casos con cavitación en la radiografía de tórax inicial y en pacientes con cultivo de esputo positivo al segundo mes de tratamiento, se propone prolongar la fase de continuación con el propósito de reducir el riesgo de recaída. La guía también recomienda retirar el etambutol una vez que se haya demostrado la sensibilidad a $H$ y $R$. Se hacen además recomendaciones de manejo en distintas situaciones clínicas como en pacientes co-infectados con VIH y en las TBC extrapulmonares. En la pericarditis TBC se sugiere no utilizar de forma rutinaria la terapia corticoesteroidal coadyuvante al tratamiento anti TBC. En el caso de la TBC meníngea se recomienda usar corticoesteroides y prolongar la quimioterapia durante la fase de continuación a 9 -12 meses.

Palabras clave: Tratamiento de la tuberculosis; Agentes antituberculosos; infección por VIH; manejo de casos; salud pública.

\footnotetext{
* Residente de Medicina Interna, Universidad Diego Portales.

** Instituto Nacional del Tórax.

***Asesores del Ministerio de Salud.
} 
La Sociedad Americana de Tórax (ATS), el Centro para el Control y Prevención de las Enfermedades Infecciosas (CDC) y la Sociedad Americana de Enfermedades Infecciosas (IDSA) patrocinaron en conjunto el desarrollo de una nueva guía de tratamiento para tuberculosis (TBC) sensible a fármacos. Esta guía ha sido respaldada por la Sociedad Respiratoria Europea (ERS) y la Asociación Nacional para el Control de la Tuberculosis (NTCA) ${ }^{1}$. Aunque el tratamiento de la tuberculosis en Chile $^{2-4}$, sigue las mismas pautas recomendadas en esta Guía, parece conveniente revisarla porque ofrecen matices útiles en su aplicación a la práctica.

Para la confección de esta Guía se utilizó el sistema P.I.C.O. (patient, intervention, comparison, outcome) para contestar 9 preguntas claves.

Pregunta 1: ¿Incluir intervenciones no farmacológicas en el tratamiento de los pacientes con $T B C$, mejora los resultados al compararlos con la terapia por sí sola?

Recomendaciones: Se sugiere realizar intervenciones no farmacológicas como educación al enfermo en relación a la infección por TBC y su tratamiento, visitas domiciliarias, coordinación de citas médicas y hospitalizaciones.

(Recomendación condicional y con evidencia baja).

El tratamiento anti TBC requiere múltiples fármacos durante varios meses, por esta razón el paciente debe estar involucrado de forma activa durante el proceso diagnóstico y terapéutico. Se proponen consideraciones claves para optimizar el manejo, entre las que se destaca la educación del enfermo sobre su patología, el tratamiento y las posibles reacciones adversas a medicamentos (RAMS). Se recomienda discutir los resultados esperados y las medidas de control de la infección con los pacientes, utilizando una terminología adecuada a su cultura, idioma y edad.

Los enfoques para asegurar la adhesión al tratamiento son un punto importante en la gestión general. Es por esto que se ha propuesto que la forma de administración de fármacos anti TBC debe ser mediante la observación directa por personal debidamente capacitado.

Pregunta 2: ¿La terapia auto-administrada tiene similares resultados en comparación con la terapia bajo observación directa en pacientes con diversas formas de TBC?
Recomendación 2: Se sugiere administrar la terapia de forma supervisada directamente para el tratamiento de rutina de pacientes en todas las formas de TBC.

(Recomendación condicional y con evidencia baja).

No se encontraron diferencias significativas entre la auto-administración y la administración con observación directa al evaluar mortalidad, término del tratamiento y recaídas; sin embargo, la observación directa se asoció de manera significativa con éxito del tratamiento (pacientes curados que completaron tratamiento) y con una mayor tasa de conversión del cultivo de esputo. Además, se ha demostrado que juega un papel importante en el reconocimiento precoz de las RAMS, en la identificación de las irregularidades de la terapia y en las posibles complicaciones del tratamiento; de esta forma ayudaría a establecer una adecuada relación con el paciente y el equipo de salud.

\section{Regímenes de tratamiento recomendados}

El régimen de terapia más adecuado y otras opciones se enumeran en la Tabla 1.

Pregunta 3: ¿La administración intermitente en la fase intensiva tiene resultados similares a los de la dosis diaria?

Recomendación 3a: Se recomienda el uso de dosis diaria en lugar de intermitente en la fase intensiva. (Recomendación fuerte con evidencia de calidad moderada).

Recomendación 3b: El uso de terapia trisemanal (con o sin dos semanas diarias iniciales) puede considerarse en pacientes que no estén infectados por VIH y que tengan bajo riesgo de recaída (sin cavitación y con baciloscopias-BK negativas).

(Recomendación condicional, con evidencia baja).

\section{Pregunta 4: ¿La dosificación intermitente versus diaria en la fase de continuación tiene resultados similares?}

Recomendación 4a: Se recomienda la administración diaria o trisemanal en la fase de continuación.

(Recomendación fuerte, con evidencia moderada). Recomendación 4b: Se sugiere la dosificación trisemanal por sobre la bisemanal en la fase de continuación.

(Recomendación fuerte, con evidencia débil). 
Tabla 1. Esquemas de tratamiento para tuberculosis pulmonar confirmada microbiológicamente causada por los organismos sensibles

\begin{tabular}{|c|c|c|c|c|c|c|}
\hline \multirow[b]{2}{*}{ Esquema } & \multicolumn{2}{|c|}{ Fase intensiva } & \multicolumn{2}{|c|}{ Fase de continuación } & \multirow[b]{2}{*}{$\begin{array}{l}\text { Total } \\
\text { dosis }\end{array}$} & \multirow[b]{2}{*}{ Comentarios } \\
\hline & Fármacos ${ }^{1}$ & $\begin{array}{l}\text { Intervalos y } \\
\text { dosis }\end{array}$ & Fármacos & $\begin{array}{l}\text { Intervalos y } \\
\text { dosis }\end{array}$ & & \\
\hline 1 & H-R-Z-E & $\begin{array}{l}7 \text { días/semana } \\
\text { por } 56 \text { dosis o } \\
5 \text { días/semana } \\
\text { por } 40 \text { dosis }\end{array}$ & H-R H-R & $\begin{array}{l}7 \text { días/semana } \\
\text { por } 126 \text { dosis o } \\
5 \text { días/semana } \\
\text { por } 90 \text { dosis }\end{array}$ & $182-130$ & $\begin{array}{l}\text { Régimen preferido para pacien- } \\
\text { tes con TBC pulmonar }\end{array}$ \\
\hline 2 & H-R-Z-E & $\begin{array}{l}7 \text { días/semana } \\
\text { por } 56 \text { dosis } \\
\text { o } 5 \text { días/semana } \\
\text { por } 40 \text { dosis }\end{array}$ & H-R & $\begin{array}{l}3 \text { días/semana } \\
\text { por } 54 \text { dosis }\end{array}$ & $110-94$ & $\begin{array}{l}\text { Esquema alternativo preferido } \\
\text { cuando la administracion super- } \\
\text { visada es compleja }\end{array}$ \\
\hline 3 & H-R-Z-E & $\begin{array}{l}3 \text { días/semana } \\
\text { por } 24 \text { dosis }\end{array}$ & $\mathrm{H}-\mathrm{R}$ & $\begin{array}{l}3 \text { días/semana } \\
\text { por } 54 \text { dosis }\end{array}$ & 78 & $\begin{array}{l}\text { Uso con precaución en pacien- } \\
\text { tes con VIH y/o enfermedad } \\
\text { cavitaria. Las dosis perdidas } \\
\text { pueden desencadenar compli- } \\
\text { caciones }^{2}\end{array}$ \\
\hline 4 & H-R-Z-E & $\begin{array}{l}7 \text { días/semana } \\
\text { por } 14 \text { dosis } \\
\text { seguido de } \\
2 \text { días/semana } \\
\text { por } 12 \text { dosis }\end{array}$ & H-R & $\begin{array}{l}2 \text { días/semana } \\
\text { por } 36 \text { dosis }\end{array}$ & 62 & $\begin{array}{l}\text { No recomendado en pacientes } \\
\text { VIH, BK (+) y enfermedad } \\
\text { cavitaria }\end{array}$ \\
\hline
\end{tabular}

${ }^{1} \mathrm{H}$ : Isoniacida, R: Rifampicina, Z: Pirazinamida, E: Etambutol. ${ }^{2}$ Las dosis perdidas pueden producir fracaso del tratamiento, recaída y adquisición de resistencia a fármacos. $\mathrm{BK}(+)$ : baciloscopia positiva.

El régimen ideal para el paciente con $\mathrm{TBC}$ sensible consiste en una fase intensiva de 2 meses de duración con isoniazida $(\mathrm{H})$, rifampicina $(\mathrm{R})$, pirazinamida $(Z)$ y etambutol (E) seguida de una fase de continuación de 4 meses de $\mathrm{H}$ y R. Con el propósito de reducir el riesgo de recaída, se recomienda extender la fase de continuación por 3 meses adicionales en pacientes con cavitación en la radiografía de tórax inicial (o de seguimiento) $y$, en aquellos cuyo cultivo del segundo mes resulte positivo.

Si la terapia se inicia después de conocer los resultados de las pruebas de sensibilidad a los fármacos y se demuestra sensibilidad a $\mathrm{H}$ y $\mathrm{R}$, el $\mathrm{E}$ no es necesario y en la fase intensiva puede darse $\mathrm{H}, \mathrm{R}$ y Z solamente. De igual forma, si ya se ha iniciado terapia con 4 fármacos, el $\mathrm{E}$ puede interrumpirse tan pronto como los resultados de pruebas de sensibilidad demuestren sensibilidad a H y R.

Además, considerando que algunos pacientes que usan $\mathrm{H}$ tienen mayor riesgo de neuropatía, como ocurre con las embarazadas, los lactantes, aquellos con coinfección por VIH, los diabéticos, los alcohólicos, aquellos con desnutrición o insuficiencia renal crónica y pacientes de edad avanzada, se recomienda el uso profiláctico de piridoxina (vitamina B6) en dosis de $25-50 \mathrm{mg} /$ día. Para los con neuropatía periférica, los expertos recomiendan aumentar la dosis de piridoxina a $100 \mathrm{mg} /$ día.

Basado en revisiones sistemáticas la frecuencia de administración del tratamiento recomendada es diaria tanto para la fase intensiva como de continuación; sin embargo, en la fase de continuación se acepta la administración trisemanal cuando la observación directa de la terapia ha resultado dificultosa.

Por otra parte, la administración de fármacos por 5 días a la semana versus 7 , no ha sido estudiada. No obstante, sobre la base de una experiencia clínica sustancial, los expertos informan que la administración de 5 días a la semana es una alternativa aceptable a la administración de 7 días a la semana, y cualquiera de los dos enfoques puede considerarse que cumple con la definición de dosificación "diaria".

\section{Regímenes alternativos de tratamiento}

En algunos casos puede existir intolerancia a fármacos de primera línea o mono-resistencia, por lo que pueden requerirse regímenes alternativos. Si la $Z$ no puede ser utilizada se recomienda el uso de HRE diario por 2 meses y posteriormen- 
te prolongar la fase continuación a 7 meses con $\mathrm{H}$ y R diario o trisemanal. En caso de no poder utilizar $\mathrm{H}$ o E, se propone en su reemplazo una fluoroquinolona (moxifloxacino o levofloxacino) en la fase intensiva y de continuación. Si no puede utilizarse $\mathrm{R}$ o varios fármacos de primera línea, se recomienda seguir las guías de tratamiento de TBC multi-resistente.

\section{Seguimiento}

Se recomienda realizar BK y cultivos mensualmente hasta que dos cultivos, en forma consecutiva resulten negativos.

La cavitación en la radiografía de tórax junto a cultivo positivo al completar dos meses de tratamiento se han asociado con mayores tasas de recaída, llegando hasta un $20 \%$ versus un $2 \%$ en pacientes sin esos factores de riesgo. En este contexto y basados en opinión de expertos se recomienda prolongar la fase de continuación con $\mathrm{H}$ y $\mathrm{R}$ por un período adicional de 3 meses.

Otros factores para prolongar el tratamiento son: presencia de cavitación pulmonar o cultivo positivo a los 2 meses de terapia (cada uno por sí sólo), $\geq 10 \%$ bajo el peso ideal, tabaquismo, diabetes mellitus, co-infección por $\mathrm{VIH}$, inmunosupresión, y alteraciones extensas en la radiografía de tórax.

Si el paciente ha estado en tratamiento durante $\geq 3$ meses y mantiene cultivos positivos, deben repetirse las pruebas de sensibilidad. Si mantiene cultivos positivos al cuarto mes de terapia se considera fracaso al tratamiento y debe ser manejado como tal.

Otro factor relevante durante el seguimiento es la adecuada pesquisa y manejo de pacientes con interrupciones de la terapia, lo cual resulta común en estos enfermos que requieren de tratamientos prolongados. Mientras más precoz y más prolongada es la interrupción, más serio será el efecto y por tanto la necesidad de reiniciar un nuevo tratamiento. En estos casos el estado bacteriológico es importante para tomar una decisión. En la fase de continuación, de mantenerse los cultivos positivos debe reiniciarse una nueva terapia; de ser negativos, se recomienda administrar $\mathrm{H}$ y $\mathrm{R}$ por 4 meses adicionales, siempre y cuando el bacilo aislado inicialmente fuera sensible y el paciente haya recibido $\mathrm{H}, \mathrm{R}$ y $\mathrm{Z}$ en la primera fase de tratamiento. Si la terapia no pudo completarse dentro del tiempo recomendado, deberá reiniciarse desde el principio (es decir, con la fase intensiva, seguida de la fase de continuación). Al reiniciar la terapia siempre deberá indicarse su administración bajo observación directa.

Los esquemas de tratamiento tras una interrupción se presentan en la Tabla 2.

Se considera terapia completa una vez que el paciente ha recibido el número total de dosis de acuerdo al programa implementado en cada país. En el caso de Chile 50 dosis en fase intensiva, seguido de 48 dosis en fase de continuación (trisemanal), completándose la fase intensiva por lo menos dentro de los primeros 3 meses, seguidos por la fase de continuación en los siguientes 4 meses.

Algunas consideraciones prácticas sobre el tratamiento incluyen la administración de la terapia en ayunas ya que optimiza la biodisponibilidad de los fármacos, recomendándose además evitar la administración de $\mathrm{H}$ con glucosa o lactosa ya que disminuye su absorción. Respecto de la presenta-

Tabla 2. Esquemas de tratamiento en interrupciones

\begin{tabular}{|c|c|c|}
\hline $\begin{array}{l}\text { Etapa de } \\
\text { interrupción }\end{array}$ & Detalles de interrupción & Propuesta de manejo \\
\hline \multirow{2}{*}{$\begin{array}{l}\text { Durante } \\
\text { fase } \\
\text { Intensiva }\end{array}$} & Lapso de interrupción es $<14$ días & $\begin{array}{l}\text { Continuar el tratamiento para completar el } \mathrm{n}^{\circ} \text { total previsto } \\
\text { de dosis (siempre y cuando se complete total en } 3 \text { meses) }\end{array}$ \\
\hline & Lapso de interrupción $>14$ días & Reinicie el tratamiento desde el principio \\
\hline \multirow{4}{*}{$\begin{array}{l}\text { Durante } \\
\text { fase } \\
\text { Continuación }\end{array}$} & $\begin{array}{l}\text { Recibió }>80 \% \text { de las dosis, con } \mathrm{BK} \\
\text { negativa en test iniciales }\end{array}$ & Puede considerarse el no continuar la terapia \\
\hline & $\begin{array}{l}\text { Recibió }>80 \% \text { de las dosis, con } \mathrm{BK} \\
\text { positiva en test iniciales }\end{array}$ & Continuar la terapia hasta completar el total de dosis \\
\hline & $\begin{array}{l}\text { Recibió }<80 \% \text { de las dosis y el lapso } \\
\text { acumulado total es }<3 \text { meses }\end{array}$ & $\begin{array}{l}\text { Continuar la terapia hasta completar dosis, a menos que } \\
\text { lapso consecutivo sea }>2 \text { meses }\end{array}$ \\
\hline & $\begin{array}{l}\text { Recibió }<80 \% \text { de las dosis y lapso } \\
>3 \text { meses }\end{array}$ & $\begin{array}{l}\text { Reiniciar la terapia desde el principio: nueva fase intensiva } \\
\text { y de continuación }\end{array}$ \\
\hline
\end{tabular}


ción de los fármacos, la administración de dosis fijas combinadas no han demostrado superioridad en sus resultados respecto de su administración por separado; sin embargo, disminuyen los errores de medicación por la mayor facilidad en su administración.

Otras consideraciones incluyen la aparición de RAM. Cuando son síntomas gastrointestinales, la recomendación es realizar una evaluación clínica y estudio de función hepática. De no demostrar hepatotoxicidad se recomienda el uso de inhibidores de la bomba de protones o su administración con una colación pequeña para mejorar la tolerancia digestiva.

En caso de hepatotoxicidad, con mayor frecuencia ocasionado por Z; H; R, en este orden, ésta puede presentarse en forma sintomática $\mathrm{o}$ asintomática. La hepatotoxicidad es definida por un aumento de las aminotransferasas $\geq 3$ veces el valor normal en presencia de síntomas digestivos $y \geq 5$ veces en casos asintomáticos. En la mayoría de pacientes asintomáticos, las pruebas hepáticas alteradas se normalizan de forma espontánea, recomendándose el control clínico y de la función hepática frecuente. De aumentar los síntomas y el deterioro de las enzimas hepáticas se recomienda la suspensión del tratamiento anti TBC. Una vez que las aminotransferasas disminuyen a 2 veces su valor normal, puede reiniciarse la quimioterapia de forma secuencial, empezando por $\mathrm{R}$ por ser menos hepatotóxica que $\mathrm{H}$ y $\mathrm{Z}$. De permanecer estable la función hepática tras 1 semana puede agregarse $\mathrm{H}$. De no presentar alteraciones tras la introducción de $\mathrm{H}$ y $\mathrm{R}$, se asume como fármaco causante de la toxicidad a la $\mathrm{Z}$ y se recomienda descontinuarla, por lo que, dependiendo de la severidad de la enfermedad y su estado bacteriológico, la duración total de la terapia podría extenderse a 9 meses.

\section{Tratamiento en situaciones especiales}

\section{Co-infección por VIH}

\author{
Pregunta 5: ¿Extender el tratamiento más allá \\ de 6 meses mejora los resultados en compara- \\ ción con el régimen de tratamiento estándar de \\ 6 meses en paciente con TBC y co-infección \\ por el VIH? \\ Recomendación 5a: Para los pacientes co-infec- \\ tados por VIH se sugiere usar el régimen diario \\ estándar de 6 meses para el tratamiento de TBC \\ pulmonar sensible a los fármacos (Recomenda- \\ ción con evidencia de muy baja certeza).
}

Recomendación 5b: En situaciones poco comunes en las que los pacientes no reciben terapia antirretroviral durante el tratamiento de la TBC, se sugiere extender la fase de continuación con Hy R por 3 meses adicionales, equivalente a un total de 9 meses de tratamiento. (Recomendación con evidencia de baja certeza).

Se recomienda realizar pruebas de VIH de rutina y consejería a todos los pacientes con diagnóstico presuntivo y demostrado de TBC. El tratamiento anti TBC en pacientes VIH tiene varias diferencias, entre las que se incluyen la necesidad de asociar terapia antirretroviral (TARV), la eventual prolongación del tratamiento y la evaluación de interacciones farmacológicas.

La mortalidad entre los pacientes con VIH y tuberculosis es alta, principalmente por complicaciones propias de la inmunosupresión y otras enfermedades oportunistas relacionadas con el VIH. La profilaxis con cotrimoxazol reduce la morbimortalidad independientemente del recuento de linfocitos CD4.

Pregunta 6: ¿Iniciar la TARV durante el tratamiento de la tuberculosis versus al final del tratamiento de la TBC mejora los resultados en los pacientes co-infectados por VIH?

Recomendación 6: Se recomienda iniciar la $T A R V$, idealmente durante las primeras 2 semanas de tratamiento de TBC para pacientes con recuento de linfocitos CD4 $<50$ células/ $\mu l y$ durante las primeras 8 a 12 semanas para pacientes con recuentos de CD4 $\geq 50$ células/ $\mu l$. Una excepción son los pacientes con VIH y meningitis tuberculosa, donde la terapia debe diferirse al menos hasta después de las 8 semanas de terapia diaria.

(Recomendación fuerte, con certeza en la evidencia).

\section{Tuberculosis extrapulmonar}

El tratamiento de la TBC extrapulmonar se inicia con $\mathrm{H}, \mathrm{R}, \mathrm{E}$ y $\mathrm{Z}$ durante una fase inicial intensiva de 2 meses, seguida de una fase de continuación de 4 a 9 meses con $\mathrm{H}$ y R, exceptuando la TBC meníngea cuya duración se recomienda prolongarla por un total de 12 meses.

\subsection{Tuberculosis ganglionar}

Se recomienda el esquema de tratamiento habitual por un total de 6 meses. La incisión qui- 
rúrgica y drenaje se ha asociado a mayor tiempo de descarga y cicatrización; sólo está reservada a casos puntuales, como linfonodos de gran tamaño y fluctuantes, y aquellos que drenen de forma espontánea.

\subsection{Tuberculosis ósea, articular y vertebral}

Se recomienda una duración de terapia de 6 a 9 meses, siempre que se incluya la $\mathrm{R}$ y 18 meses en el caso de no incluirla. Basado en opinión de expertos, se plantea realizar manejo quirúrgico en casos de pobre respuesta a terapia anti TBC, con deterioro clínico y persistencia o recurrencia de déficits neurológicos e inestabilidad espinal.

\subsection{Pericarditis tuberculosa}

Se recomienda la administración de terapia anti TBC por 6 meses.

Pregunta 7: ¿El uso de corticoesteroides de forma concomitante a la terapia anti TBC en pacientes con pericarditis TBC ha demostrado beneficios en la morbimortalidad?

Recomendación 7: No se recomienda el uso de corticoesteroides en conjunto con la terapia anti $T B C$ de forma rutinaria.

(Recomendación condicional, con baja evidencia).

Anteriormente, el uso de corticoides en conjunto con la terapia anti-TBC era una indicación universal; sin embargo, en la actualidad se ha demostrado que su empleo no aporta beneficios en mortalidad, tamponamiento cardíaco y desarrollo de pericarditis constrictiva. Por lo tanto, la indicación de corticoesteroides se reserva para pacientes con alto riesgo de complicaciones inflamatorias, como un derrame pericárdico severo, altos niveles de marcadores y/o de células inflamatorias en el líquido pericárdico y signos tempranos de pericarditis constrictiva.

\subsection{Tuberculosis pleural}

Se recomienda régimen de terapia por 6 meses. El uso rutinario de corticoesteroides no ha demostrado beneficios. En el caso de empiema tuberculoso, existe un gran número de bacilos en el espacio pleural, lo que habitualmente ocurre por ruptura de una cavidad pulmonar al espacio pleural. El tratamiento consiste en drenaje (pudiendo requerir manejo quirúrgico) asociado a la terapia farmacológica, cuya duración óptima no ha sido establecida.

\subsection{Tuberculosis meníngea}

Pregunta 8: ¿El uso de corticoesteroides adyuvantes en meningitis tuberculosa proporcionan beneficios en la mortalidad y morbilidad?

Recomendación 8: Se recomienda el uso de corticoesteroides desde el inicio, en conjunto con la terapia anti TBC con dexametasona o prednisolona por 6 a 8 semanas en pacientes con meningitis tuberculosa.

(Recomendación con certeza moderada en la evidencia).

La meningitis tuberculosa sigue siendo una enfermedad grave y con alta morbimortalidad aunque haya un inicio precoz del tratamiento. Los pacientes co-infectados por VIH tienen mayor riesgo para el desarrollo de meningitis tuberculosa, que tiene en ellos características clínicas similares.

La terapia para meningitis tuberculosa se inicia con $\mathrm{H}, \mathrm{R}, \mathrm{Z}$ y $\mathrm{E}$ en una fase inicial de 2 meses, seguida de una de continuación con $\mathrm{H}$ y $\mathrm{R}$ por un período adicional de 7 a 10 meses, aunque la duración de la terapia no ha sido establecida. De acuerdo a la opinión de expertos, debería considerarse el monitoreo del líquido cefalorraquídeo para evaluar los cambios en el recuento de células, glucosa y proteínas durante el tratamiento.

En niños con meningitis tuberculosa se recomienda un régimen inicial de 4 fármacos: $\mathrm{H}, \mathrm{R}, \mathrm{Z}$ y etionamida en lugar de $\mathrm{E}$, o un aminoglucósido durante 2 meses, seguido de 7 a 10 meses de $\mathrm{H}$ y R. El uso de corticoesteroides adyuvantes a la terapia anti TBC ha demostrado beneficios en la morbi-mortalidad, por lo que se recomienda su empleo por 6 a 8 semanas.

La neurocirugía debe considerarse en casos de meningitis tuberculosa complicada como hidrocefalia, absceso cerebral tuberculoso y situaciones clínicas en las que exista paraparesia.

\subsection{Tuberculosis diseminada}

Basado en la opinión de expertos, se recomienda un régimen diario estándar de 6 meses de terapia para la $\mathrm{TBC}$ en múltiples órganos y para la TBC miliar; sin embargo, los datos son limitados en este tipo de presentación. Algunos expertos recomiendan el agregado de corticoesteroides para el tratamiento de insuficiencia respiratoria e insuficiencia suprarrenal causadas por la TBC diseminada, aunque su papel en TBC miliar aún es incierto. 


\subsection{Tuberculosis Génito-urinaria}

Para la tuberculosis renal se recomienda un régimen de terapia por 6 meses, con ajuste de dosis de acuerdo a la función renal. El cultivo de orina positivo para $M$. tuberculosis es un componente diagnóstico necesario. En el caso de cursar con obstrucción ureteral, hidronefrosis o insuficiencia renal progresiva, pueden estar indicados procedimientos quirúrgicos.

\subsection{Tuberculosis abdominal}

Se recomienda un régimen de 6 meses de terapia para pacientes con tuberculosis peritoneal o intestinal. La presentación clínica es habitualmente inespecífica por lo que requiere un alto índice de sospecha para un diagnóstico y tratamiento precoz. La terapia adyuvante con corticoesteroides no está indicada de forma rutinaria; los datos sobre sus beneficios son limitados.

\subsection{Tuberculosis pulmonar con cultivos negativos}

Pregunta 9: ¿Podría realizarse un tratamiento de menor duración en los pacientes no infectados VIH con tuberculosis paucibacilar, es decir, con BK negativa y cultivo negativo?

Recomendación 9: Se recomienda un régimen de tratamiento de 4 meses en pacientes adultos no infectados por VIH con BKy cultivos negativos. (Recomendación con evidencia de muy baja certeza).

En caso de sospecha clínica o radiológica de TBC pulmonar, pero con BK y cultivos negativos, el diagnóstico no debe ser excluido, aunque deben considerarse diagnósticos diferenciales.

Algunas causas de fracaso para aislar organismos incluyen: el uso reciente de antibióticos con actividad bactericida contra $M$. tuberculosis (por ejemplo, fluoroquinolonas); poblaciones bacilares bajas; muestras de esputo inadecuadas; variaciones temporales en el número de bacilos expulsados; crecimiento excesivo de otros microorganismos, y errores en el procesamiento de las muestras.

En todo paciente con sospecha de TBC pulmonar deben realizarse $2 \mathrm{BK}$. De no ser posible, por falta de expectoración, esta puede inducirse mediante nebulización con solución salina hipertónica para obtener muestras para BK y cultivos. Otros métodos diagnósticos incluyen la fibrobroncoscopía con lavado broncoalveolar y biopsia.

En un paciente con sospecha clínica o radioló- gica de TBC pulmonar, debería iniciarse terapia anti TBC con un régimen de 4 fármacos, incluidos H, R, Z y E pese a tener cultivos de esputo iniciales negativos. Si se aísla $M$. tuberculosis en un cultivo de esputo o mediante una prueba molecular rápida, se continúa la terapia hasta completarla con un régimen estándar de 6 meses, basándose en las pruebas de susceptibilidad a fármacos. En el caso de pacientes con cultivos de esputo negativos, pero con alta sospecha de TBC pulmonar, debe iniciarse terapia estándar y realizarse un seguimiento clínico y radiológico seriado. Si después de 2 meses de terapia existe mejoría clínica y/o radiológica y no se identifica otra etiología, debe continuar el tratamiento.

Basado en revisiones de ensayos clínicos, se recomienda una terapia de sólo 4 meses de duración en pacientes adultos con sospecha de TBC pulmonar y cultivos de esputo negativos, con una fase inicial intensiva de 4 fármacos durante 2 meses, seguida de una fase de continuación con $\mathrm{H}$ y R por 2 meses más. Debe evaluarse la respuesta clínica y/o radiológica al final de los 4 meses para determinar si debiera continuarse la terapia hasta completar 6 meses. Cuando existe duda sobre la negatividad de los estudios microbiológicos, es preferible completar un régimen estándar de 6 meses.

\section{Conclusiones}

El tratamiento actual de la TBC tiene dos objetivos principales: Curar al paciente afectado $y$ minimizar la transmisión del M. tuberculosis en la comunidad. Es importante también prevenir la aparición de resistencia bacteriana. Para ello el inicio de la terapia debe ser lo más precoz posible y bajo observación directa.

Uno de los principales aportes de esta guía es brindar distintos esquemas de terapia de acuerdo a la condición del paciente, proporcionando recomendaciones sobre el esquema ideal de manejo, iniciando una fase intensiva de 2 meses con 4 fármacos, pudiendo retirar el etambutol si se demuestra sensibilidad a $\mathrm{H}$ y $\mathrm{R}$, siguiendo con una fase de continuación por 4 meses (completando el número de dosis propuestas). Además, proporciona distintas recomendaciones de tratamiento ante condiciones especiales como TBC y co-infección por VIH y TBC extrapulmonar, que son bastante similares a las nuestras.

De acuerdo a las normas técnicas de tratamiento anti TBC vigentes en Chile, actualmente cumplimos con las principales recomendaciones dictadas en esta guía. Consideramos como una 
alta recomendación la educación del paciente y utilizamos el esquema de tratamiento con una fase intensiva con dosis diarias (5 días/semana) de $\mathrm{H}, \mathrm{R}, \mathrm{Z}$ y E seguida de una fase de continuación con administración trisemanal de $\mathrm{H}$ y $\mathrm{R}$, dándole especial importancia al tratamiento totalmente supervisado.

Generalmente mantenemos el etambutol en la fase diaria ya que nuestras pruebas de susceptibilidad dan resultados tardíos, No reemplazamos el etambutol por moxifloxacino, reservando este fármaco para pacientes multirresistentes (TBMDR). No tratamos los casos monoresistentes a $\mathrm{R}$ como TB-MDR si son sensibles a $\mathrm{H}$, ni prolongamos la terapia en fumadores ni diabéticos. No usamos etionamida en la meningitis infantil y no reducimos el tratamiento a sólo 4 meses en los casos sin comprobación bacteriológica, para asegurarnos de evitar recaídas.

Dentro de los factores de riesgo conocidos de recaída se encuentran la cavitación pulmonar y el cultivo positivo al completar 2 meses de tratamiento y los pacientes co-infectados por VIH. En algunos de estos casos se propone prolongar la terapia en su fase de continuación, para completar 9 meses en total, lo que también recomendamos nosotros.

En cambio, en pacientes con fuerte sospecha clínico/radiológica de TBC pulmonar debería iniciarse el tratamiento sin esperar el resultado de $\mathrm{BK}$, pruebas moleculares y/o cultivos de esputo.

Considerando los bajos niveles de evidencia en los que se basan todas las guías internacionales, creemos que los países deben considerar sus propias experiencias para decidir las adaptaciones terapéuticas más convenientes para sus pacientes.

\section{Bibliografía}

1.- NAHID P, DORMAN SE, ALIPANAH N, BARRY PM, BROZEK JL, CATTAMANCHI A, et al. Official American Thoracic Society/Centers for Disease Control and Prevention/Infectious Diseases Society of America Clinical Practice Guidelines: Treatment of Drug-Susceptible Tuberculosis. Clin Infect Dis 2016; 63 (7): e147-e195. DOI: https://doi.org/10.1093/cid/ciw376. Disponible en: https://academic.oup.com/cid/article/63/7/e147/2196792/ Official-American-Thoracic-Society-Centers-for (Consultado el 24 de marzo de 2017).

2.- MINISTERIO DE SALUD. Manual de Organización y Normas Técnicas. Programa Nacional de Control de la Tuberculosis. 2005. Santiago. Chile.

3.- MINISTERIO DE SALUD. Programa Nacional de Control y Eliminación de la Tuberculosis. Informe de situación. 2012. Santiago. Chile.

4.- FARGA V. Cambios en el tratamiento de la tuberculosis. Rev Chil Enferm Respir 2014; 30: 174-9. 